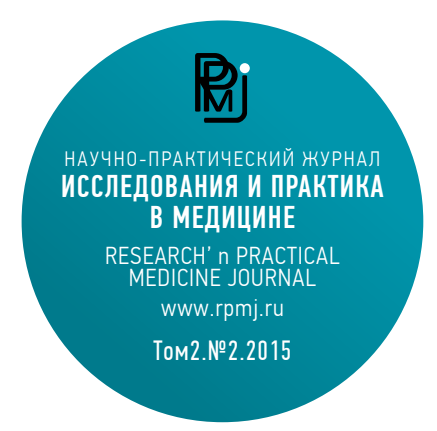

Ключевые слова:

опухоли костей,

эндопротезирование,

функциональный результат

Keywords:

bone tumors,

endoprosthetic replacement,

functional result

DOI: 10.17709/2409-2231-2015-2-2-15-23

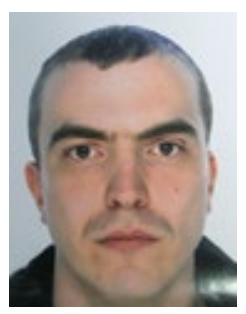

Для корреспонденции:

Державин Виталий Андреевич,

к.М.Н., научный сотрудник группы опухолей костей и мягких тканей, отдела хирургического лечения опухолей центральной нервной и костно-мышечной систем МНИОИ им. П.А. Герцена - филиал ФГБУ «НМИРЦ» Минздрава России

Адрес: 125284 , Российская Федерация, Москва, 2-ой Боткинский проезд, д. 3.

E-mail: osteosa@yandex.ru

Статья поступила 28.04.2015,

принята к печати 11.06.2015

For correspondence:

Derzhavin Vitaly - PhD,

senior researcher of group of bone and soft

tissue tumors, division of surgical treatment

of tumors of the central nervous

and musculoskeletal systems

P. Hertsen MORI - branch of NMRRC

Address: 2-y Botkinskiy proezd, 3, Moskva

E-mail: osteosa@yandex.ru

The article was received 28.04.2015

accepted for publication 11.06 .2015

\section{ЭНДОПРОТЕЗИРОВАНИЕ ПЛЕЧЕВОГО СУСТАВА ПРИ ОПУХОЛЕВОМ ПОРАЖЕНИИ ПРОКСИМАЛЬНОГО ОТДЕЛА ПЛЕЧЕВОЙ КОСТИ}

Карпенко В.Ю., Державин В.А., Бухаров А.В.

МНИОИ им. П.А. Герцена - филиал ФГБУ «НМИРЦ» Минздрава России

125284, Российская Федерация, г. Москва, 2-ой Боткинский проезд, дом 3

\section{Резюме:}

Введение. В настоящее время у $90 \%$ пациентов со злокачественным поражением длинных костей и крупных суставов возможно органосохранное хирургическое лечение - онкологическое эндопротезирование. Благодаря развитию и совершенствованию хирургической техники, применению новейших антибактериальных препаратов, разработке новых систем модульного эндопротезирования удалось добиться значимого снижения осложнений, таких как инфицирование, нестабильность эндопротеза, а также получить хорошие и отличные функциональные результаты после операции, в основном при поражении костей нижних конечностей. Риск развития осложнений при проведении онкологического эндопротезирования верхней конечности также невысок, однако функциональные показатели после этих операций существенно ниже.

Цель. Проанализировать и оценить результаты применения «Анатомических» и "Обратных» эндопротезов плечевого сустава у больных с опухолевым поражением проксимального отдела плечевой кости.

Материалы и методы. В зависимости от типа установленного металлоимпланта больные были разделены на две группы. "Анатомический» эндопротез был установлен 28 пациентам (13 мужчин и 15 женщин), а «Обратный» эндопротез 14 больным (6 мүжчин и 8 женщин). Средней возраст составил в первой группе $32 \pm 4$ лет и во второй $38 \pm 3$ лет соответственно. Первичные опухоли костей были диагностированы у 19 (68\%) больных первой и 12 (86\%) пациентов второй группы.

Результаты. Средний срок наблюдения составил $42 \mathrm{mеc.} \mathrm{Прогрессирование}$ заболевания в сроки от 6 до 14 мес. было у 4-х больных с первичными злокачественными опухолями костей. Метастатическое поражение легких выявлено у 2-х из них, рецидив опухоли - у одной больной и рецидив с метастатическим поражением легких также у одной больной. В группе больных с "Анатомическим» эндопротезированием плечевого сустава функциональный статус после операции был оценен по шкале MSTS (Musculo skeletal tumor standing System) в диапазоне от 60-80 баллов - у 3-х, 4060 баллов - у 6-ти и менее 40 баллов - у 19 больных соответственно. Пациентам, которым установлен “Обратный» эндопротез функциональный статус по шкале MSTS находился в диапазоне от 80 до 100 баллов у 6-ти больных, 60-80 баллов - у 6-ти, 40-60 баллов - у 2-х больных соответственно. Отмечено улучшение функционального статуса при «Обратном» эндопротезировании на $75 \%$.

Заключение. Использование модульных «Обратных» эндопротезов в органосохранном лечении больных с опухолевым поражением проксимального отдела плечевой кости, по сравнению с "Анатомическим» эндопротезированием, является более перспективной методикой, т. к. позволяет значимо улучшить функциональный результат и социальную адаптацию после операции. 


\section{ENDOPROSTHETIC REPLACEMENT IN PATIENTS WITH TUMORS OF PROXIMAL HUMERUS}

Karpenko V.Yu., Derzhavin V.A., Buharov A.V.

P. Hertsen MORI

2-y Botkinskiy proezd, d.3, Moskva, 125284, Russia

\section{Abstract}

Introduction Currently the organ-preserving surgical treatment (oncological arthroplasty) may be provided in $90 \%$ of patients with malignant lesions of the long bones and large joints. Due to the development and improvement of surgical techniques, the application of new antibacterial drugs, the development of new systems of modular endoprosthesis managed to achieve a meaningful reduction of complications, such as infection, instability of the endoprosthesis, and receive good and excellent functional results after surgery, mainly in lesions of the bones of the lower extremities. The risk of complications in conducting oncoloicaly arthroplasty upper extremity is also low, but functional performance after these operations is significantly lower.

Objective. To analyze and evaluate the results of applying the "Anatomical» and "Reverse» shoulder replacements in patients with neoplastic lesions of the proximal humerus.

Materials and methods. Depending on the type of metal implants patients were divided into two groups. "Anatomical» implant was installed in 28 patients ( 13 men and 15 women) and "Reverse» prosthesis in 14 patients ( 6 men and 8 women). The average age was in the first group $32 \pm 4$ years and the second $38 \pm 3$ years, respectively. Primary bone tumors were diagnosed in $19(68 \%)$ patients first and $12(86 \%)$ patients of the second group.
Results. The average follow-up period was 42 months. 4 patients with primary malignant bone tumors had the progression of the disease during the period from 6 to 14 months. In 2 of them they diagnosed the metastatic lung disease, in one case - the recurrence of tumor and also in one patient there was the relapse with metastases to the lungs. In the group of patients with "Anatomical» arthroplasty of the shoulder joint functional status after surgery was assessed according to the scale MSTS (Musculoskeletal tumor standing System) in the range of $60-80$ points - 3, 40-60 points -6 and less than 40 points in 19 patients, respectively. Patients who selected «Reverse» prosthesis of functional status on a scale MSTS were in the range of from 80 to 100 points in 6 patients , $60-80$ points $-6,40-60$ points - 2 patients, respectively. They marked the improvement in functional status with Reverse arthroplasty in $75 \%$ of cases.

Conclusion. The use of modular "Return" implants in organ-preserving treatment of patients with neoplastic lesions of the proximal humerus, compared to the "Anatomical» arthroplasty, is a more promising technique, because it allows significantly improved functional outcome and social adaptation after surgery.

\section{Введение}

Первичные злокачественные опухоли костей составляют от 0,2 до $2,0 \%$ от всех злокачественных новообразований [1]. Морфологически наиболее часто встречаются: остеосаркома (55-63\%), хондросаркома (17-25\%) и опухоли семейства саркомы Юинга (8-15\%) [2,3]. Также в кости часто метастазируют другие солидные опухоли. Поражение скелета выявляется у 65-73\% больных раком молочной железы, у 56-68\% раком предстательной железы и у $30-36 \%$ раком легкого $[4,5,6]$. Первичные злокачественные и метастатические опухоли костей могут поражать любой сегмент скелета, но наиболее часто локализуются в метадиафизах бедренной, плечевой и большеберцовой костей $[7,8]$. Основными клиническими проявлениями опухолевого поражения костей являются болевой синдром, нарушение функции конечности и как следствие, снижение качества жизни больного.

До семидесятых годов прошлого столетия при опухолевом поражении костей конечностей операциями выбора являлись ампутация или экзартикуляция [9]. Благодаря совершенствованию хирургической техники, развитию анестезиологического пособия и антибактериальной терапии, пациентам с опухолевым поражением длинных костей стало возможным проведение органосохранного хирургического лечения с использованием металлоимплантов. В современной онкоортопедии стандартом хирургического лечения этой категории больных является проведение сегментарной резекции кости с онкологическим эндопротезированием $[2,10,11]$. При выполнении подобных операций на костях и суставах нижней конечности частота возникновения значимых послеоперационных осложнений, таких как инфицирование или вывих эндопротеза и развитие асептической нестабильности не превышает 5-10\%, и у большинства больных после операции удается достичь адекватного функционального результата и социальной адаптации $[2,12,13,14,15]$. При опухолевом поражении длинных костей верхней конечности риск развития послеоперационных осложнений также невысок и не превышает $3 \%$, однако функциональные результаты значимо ниже [2]. Основные причины включают частую травматизацию подмышечного нерва в стремлении достигнуть максимального радикализма операции, удаление в блоке с опухолью массива отводящих мышц плечевого сустава с последующим нарушением их функции, недостаточную конгруэнтность головки эндопротеза поверхности плечевого отростка лопатки $[16,17,18]$. До настоящего времени при онкологическом эндопротезировании плечевого сустава применялись так называемые «ана- 
томические» металлоимпланты, головка которых фиксировалась к плечевому отростку лопатки и акромиону при помощи биосинтетической манжеты, к которой реимплантировались фрагменты резецированных мышц (рис. 1).

Подобная методика позволяла добиться адекватной стабильности в оперированной конечности, однако у большинства больных после операции наблюдалось значимое ограничение отведения и поднятия оперированной конечности в плечевом суставе из-за недостаточной конгруэнтности головки эндопротеза и суставной поверхности плечевого отростка лопатки $[2,18]$.

Изобретение и внедрение в ортопедию и онкоортопедию модифицированной «Обратной» конструкции эндопротеза плечевого сустава со стабилизированной суставной площадкой, которая фиксируется к плечевому отростку лопатки, и суставной впадиной, находящейся на головке эндопротеза, позволило добиться в послеоперационном периоде не только необходимой стабильности искусственного сустава, но и значимо повысить мобильность оперированной конечности в нем $[19,20]$ (рис. 2).

Вышеизложенное свидетельствует о перспективности использования в онкоортопедии «Обратных» эн- допротезов плечевого сустава как метода локального контроля в плане хирургического и комбинированного лечения пациентов с опухолевым поражением плечевой кости, позволяющего достичь адекватных онкологических и функциональных результатов.

\section{Материалы и методы}

С января 2006 года по июнь 2014 года в ФГБУ Московском Научно-исследовательском Онкологическом институте имени П. А. Герцена Минздрава России хирургическое лечение в объеме проксимальной резекции плечевой кости с эндопротезированием плечевого сустава проведено 42 пациентам с опухолевым поражением проксимального отдела плечевой кости. В зависимости от типа установленного металлоимпланта больные были разделены на две группы. «Анатомический» эндопротез (АЭ) был установлен 28 пациентам (13 мужчин и 15 женщин), а «Обратный» эндопротез (ОЭ) 14 больным (6 мужчин и 8 женщин). Средней возраст составил в первой группе $32 \pm 4$ лет и во второй $38 \pm 3$ лет соответственно. Первичные опухоли костей были диагностированы у 19 (68\%) больных первой и 12 (86\%) пациентов второй группы. Распределение больных в зависимости от морфоло-

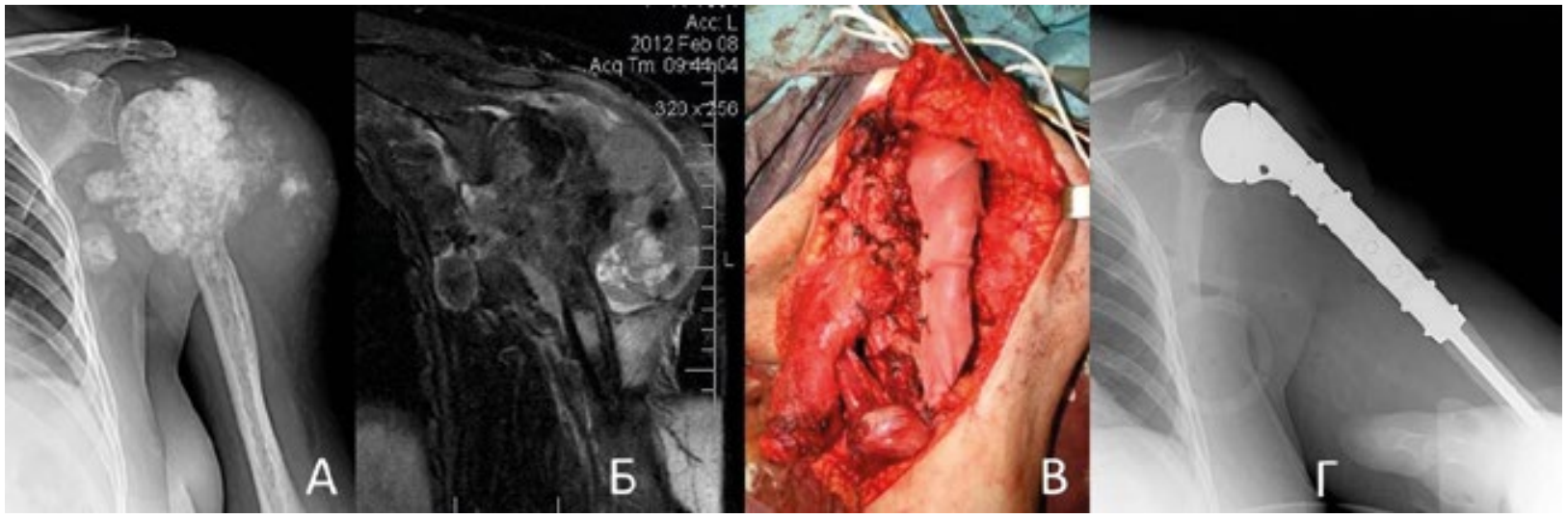

Рисунок 1. А - Рентгенограмма; Б - МРТ-грамма плечевой кости до операции (остеосаркома T2NOMO G-II IIb cт.); B - Интраоперационный вид раны (эндопротез укрыт биосинтетической манжетой); Г- Рентгенограмма на 2-ые сутки после операции

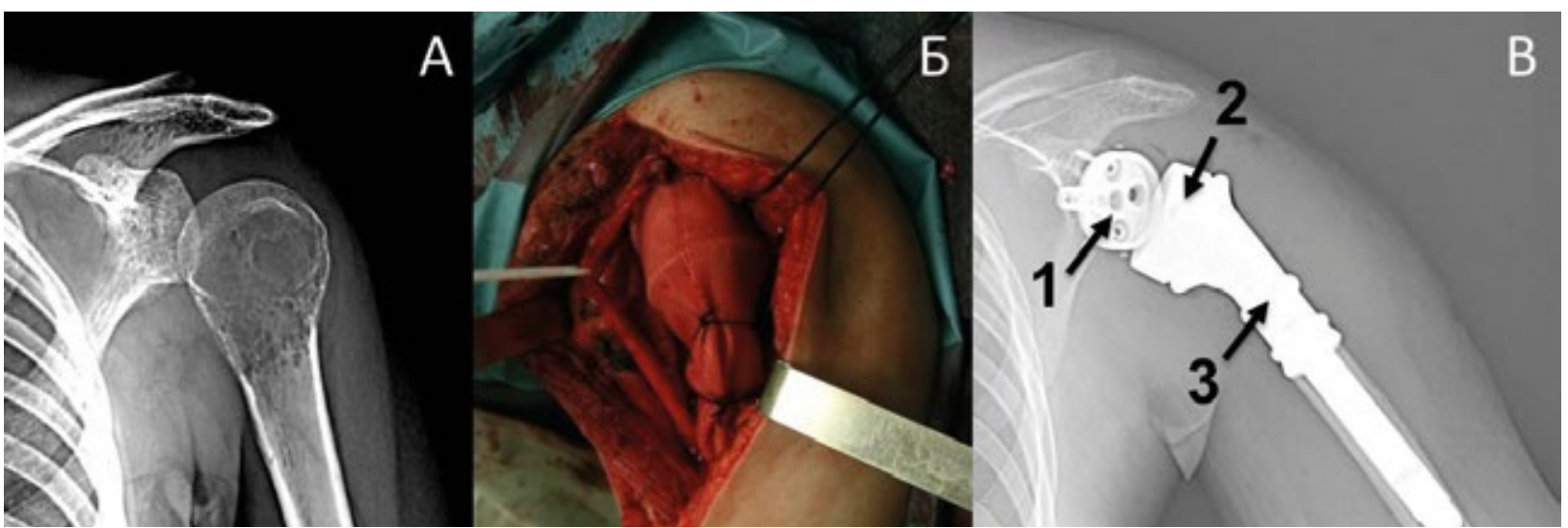

Рисунок 2. А - Рентгенограмма, плечевой кости до операции (гигантоклеточная опухоль); Б - Интраоперационный вид раны (эндопротез укрыт биосинтетической манжетой); В - Рентгенограмма на 2-ые сутки после операции (1 - фиксированная к плечевому отростку лопатки суставная площадка, 2 - суставная впадина, 3 - резекционная часть) 
гической принадлежности опухоли и типа выполненной операции приведено в таблице 1.

У больных первичными злокачественными опухолями в первой группе клиническая стадия процесса установлена как la - у 6-ти, lb - у 2-x, Ila - у одного и llb у 5-ти пациентов соответственно. Во второй группе распределение по стадиям было следующим: la - у 2-х, $\mathrm{lb}-$ у 2-x, Ila - у одного и Ilb - у 4-х больных.

Необходимо отметить, что хирургическое лечение по поводу метастатического поражения плечевой кости проводилось только при солитарном поражении.

На этапе предоперационного планирования всем пациентам проводилась компьютерная томография (КT) легких, ультразвуковое исследование брюшной полости, печени, регионарных зон, области поражения и сосудов нижних конечностей, остеосцинтиграфия, стандартное рентгеновское исследование (СР) пораженной плечевой кости в двух проекциях. При наличии клинических признаков вовлечения магистрального сосудисто-нервного пучка выполнялась магнитно-резонансная томография (МРТ). У всех пациентов с первичными злокачественными опухолями костей перед операцией выполнялась морфо- логическая верификация. У 8-ми пациентов с метастатическим поражением плечевой кости опухолевый процесс верифицирован морфологически и у 3-х - цитологически.

у больных с первичными злокачественными опухолями костей в зависимости от степени дифференцировки (G) вырабатывался план лечения. Так, всем больным с первичными опухолями низкой степени злокачественности (GI) выполнено только хирургическое лечение. Пациентам c G II и GIII проведено комбинированное лечение согласно протоколам, утвержденным в МНИОИ им. П. А. Герцена.

Все оперативные вмешательства проводились под интубационным наркозом. У всех пациентов первой группы был реализован передний дельто-пекторальный хирургический доступ и выполнены внутрисуставные резекции с отсечением сухожилия дельтовидной мышцы у места прикрепления к плечевой кости. В группе больных с «Обратным» эндопротезированием у 6-ти пациентов (43\%) применялся модифицированный заднемедиальный доступ Campbell -Anderssen. Этот хирургический доступ при небольших объемах мягкотканного компонента опухоли, по нашему мнению, с учетом всех онкологических принципов, позволяет более экономно выполнять резекцию

Таблица 1

Распределение очагов поражения плечевой кости в зависимости от морфологической принадлежности

\begin{tabular}{l|l|l|l}
\hline Морфологическая форма & АЭ & ОЭ & Всего (\%) \\
\hline Остеосаркома & 5 & 4 & $9(22)$ \\
\hline Хондросаркома & 8 & 5 & $13(31)$ \\
\hline Злокачественная фиброзная гистиоцитома & 1 & - & $1(2)$ \\
\hline Гигантоклеточная опухоль & 5 & 3 & $8(20)$ \\
\hline Метастаз рака молочной железы & 3 & - & $3(6)$ \\
\hline Лимфопролиферативные заболевания & 1 & - & $1(2)$ \\
\hline Метастаз рака почки & 4 & 2 & $6(15)$ \\
\hline Метастаз рака легкого & 1 & - & $1(2)$ \\
\hline Всего & 28 & 14 & $42(100)$ \\
\hline
\end{tabular}

Таблица 2.

Объем резекции дельтовидной мышцы

\begin{tabular}{l|l|l|l}
\hline Объем резекции & АЭ (\%) & ОЭ (\%) & Всего (\%) \\
\hline Только иссечение области биопсии & $12(42)$ & $6(44)$ & $18(43)$ \\
\hline Плечевой пучок & $8(27)$ & $4(28)$ & $12(29)$ \\
\hline Лопаточный пучок & $1(4)$ & - & $1(2)$ \\
\hline Ключичный и плечевой пучки & $2(8)$ & $1(7)$ & $3(7)$ \\
\hline Плечевой и лопаточный пучок & $2(8)$ & $2(14)$ & $4(9,5)$ \\
\hline Субтотальная резекция & $3(11)$ & $1(7)$ & $4(9,5)$ \\
\hline Всего: & $28(100)$ & $14(100)$ & $42(100)$ \\
\hline
\end{tabular}


дельтовидной мышцы плеча, сохранить ее иннервацию и прикрепление к плечевой кости [21]. Объем резекции дельтовидной мышцы представлен в таблице 2.

У наибольшего количества пациентов в первой (42\%) и второй (44\%) группах иссечение волокон дельтовидной мышцы было минимальным. Резекция плечевого пучка дельтовидной мышцы была выполнена у меньшего количества больных обеих групп, $27 \%$ и $28 \%$ соответственно. Дельтовидная мышца была резецирована субтотально у 11\% больных первой и 7\% пациентов второй группы. Пластическое формирование ложа эндопротеза перемещенным торакодорзальным лоскутом было проведено у одного больного второй группы. В целом показатели выполненных операций в зависимости от степени резекции дельтовидной мышцы в обеих группах были сопоставимы.

Уровень резекции определяли на этапе предоперационного планирования по результатам КТ, СР и МРТ. Средняя протяженность резекции составила 100 мм от оси вращения сустава. После удаления макропрепарата с опила плечевой кости осуществлялся забор костного мозга для срочного цитологического исследования и определения радикальности костной резекции. Для придания стабильности и лучшего формирования ложа эндопротеза у всех пациентов эндопротез был укрыт биосинтетической манжетой, к которой подшивали края резецированных мышц. Рана ушивалась послойно, в ложе эндопротеза оставляли дренаж на 1-2 суток. В послеоперационном периоде назначалась профилактическая антикоагулянтная, антибактериальная и сим- птоматическая терапия. Все больные были активизированы на 1-2 сутки после операции. Функциональная реабилитация больных производилась соответственно установленному типу импланта. При имплантации АЭ оперированная конечность фиксировалась отводящей шиной на срок до 2-3 мес. После установки ОЭ иммобилизация конечности шиной проводилась до 23-30 дней, после чего шина удалялась. Назначалась лечебная физкультура и специальная гимнастика для предотвращения развития мышечных контрактур и достижения адекватного функционального результата.

После окончания лечения пациент находится под динамическим наблюдением. Первые три года кратность проведения контрольного обследования для выявления возможного прогрессирования заболевания определяется интервалом в 3 месяца, в следующие 5 лет каждые 6 месяцев, далее один раз в год

\section{Результаты}

Функциональные результаты после операции оценивались по шкале MSTS [22]. Для удобства восприятия показатель MSTS в диапазоне 80-100 баллов был принят за отличный функциональный результат, 60-80 баллов за хороший, 40-60 баллов за удовлетворительный и менее 40 баллов за неудовлетворительный результат. Функциональные показатели обеих групп больных после операции представлены на рисунке 3.

Анализ данных, представленных на рисунке 3, показывает, что у большинства пациентов, которым проведе-

$\%$
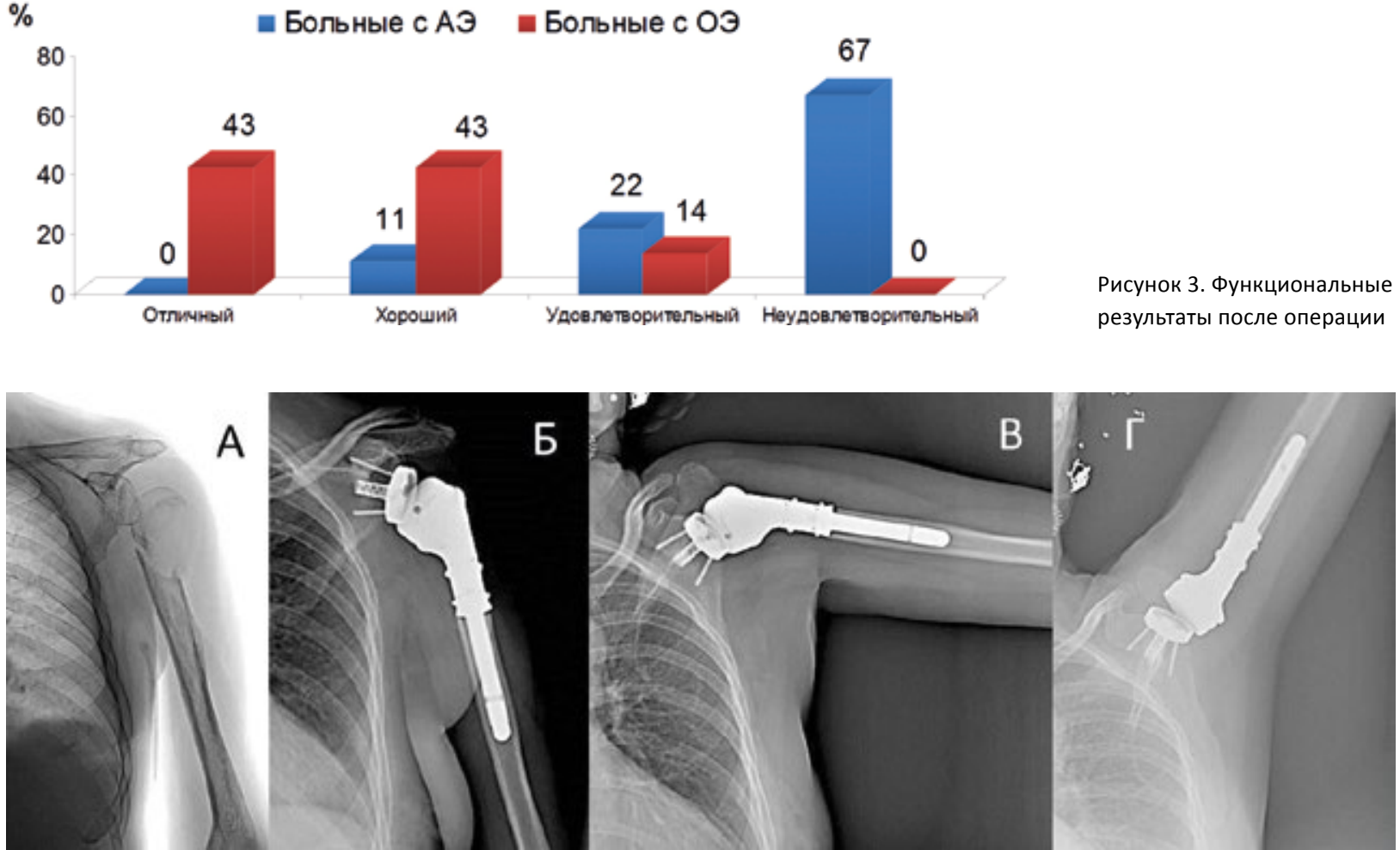

Рисунок 4. А - Рентгенограмма, плечевой кости до операции (солитарный метастаз рака почки в проксимальный отдел плечевой кости); Б-Г - Рентгенограммы через месяц после операции 
но ОЭ плечевого сустава, удалось достигнуть отличного (43\%) и хорошего (43\%) функциональных результатов. У больных после АЭ отличного результата достичь не удалось, а показатели хорошего функционального результата были существенно ниже - 11\%. В целом отмечено, что улучшение функционального статуса больных после операции при выполнении ОЭ на 75\% выше в сравнении с больными, которым был установлен АЭ (рис. 4,5).

\section{Онкологические результать}

Под наблюдением находилось 42 пациента, больных выбывших из-под наблюдения не было. По результатам планового морфологического исследования у одного больного после АЭ диагностирован положительный (индекс R1) край резекции опухоли по мягким тканям. У всех остальных пациентов обеих групп операции были радикальными с индексом RO.

Средний период наблюдения в первой группе (АЭ) составил 26 мес. (14-82 мес.). За это время прогрессирование заболевания диагностировано у 6-ти (22\%) больных, из которых у 5-ти диагностировано метастатическое поражение легких, и у одной больной местный рецидив, по поводу которого проведено хирургическое лечение в объеме межлопаточно-грудного вычленения. За время наблюдения умерло 4 больных от прогрессирования заболевания.
Средняя продолжительность наблюдения во второй группе составила 10 мес. (6-24 мес.). Прогрессирование процесса выявлено у 3-х (21\%) больных. У двух пациентов диагностированы метастазы в легкие, и у одного - местный рецидив с метастатическим поражением легких. За период наблюдения скончался один больной от прогрессирования заболевания.

Небольшое количество пациентов и непродолжительные сроки наблюдения на настоящий момент не позволяют сделать категоричных заключений. Однако при анализе имеющихся данных существенных различий при оценке онкологических результатов между пациентами с АЭ и ОЭ не получено.

\section{Осложнения}

Осложнения в послеоперационном периоде диагностированы у 2-х (7\%) пациентов первой группы. Осложнения включали инфицирование ложа эндопротеза и вывих головки эндопротеза. Больному с инфицированным ложем эндопротеза металлоимплант был удален. Реэндопротезирование не проводилось ввиду наличия хронической инфекции в ложе металлоимпланта по результатам бактериологического исследования на протяжении 14 месяцев после удаления эндопротеза. Больному с вывихом головки эндопротеза корригирующее хирургическое лечение также не выполнялось, ввиду

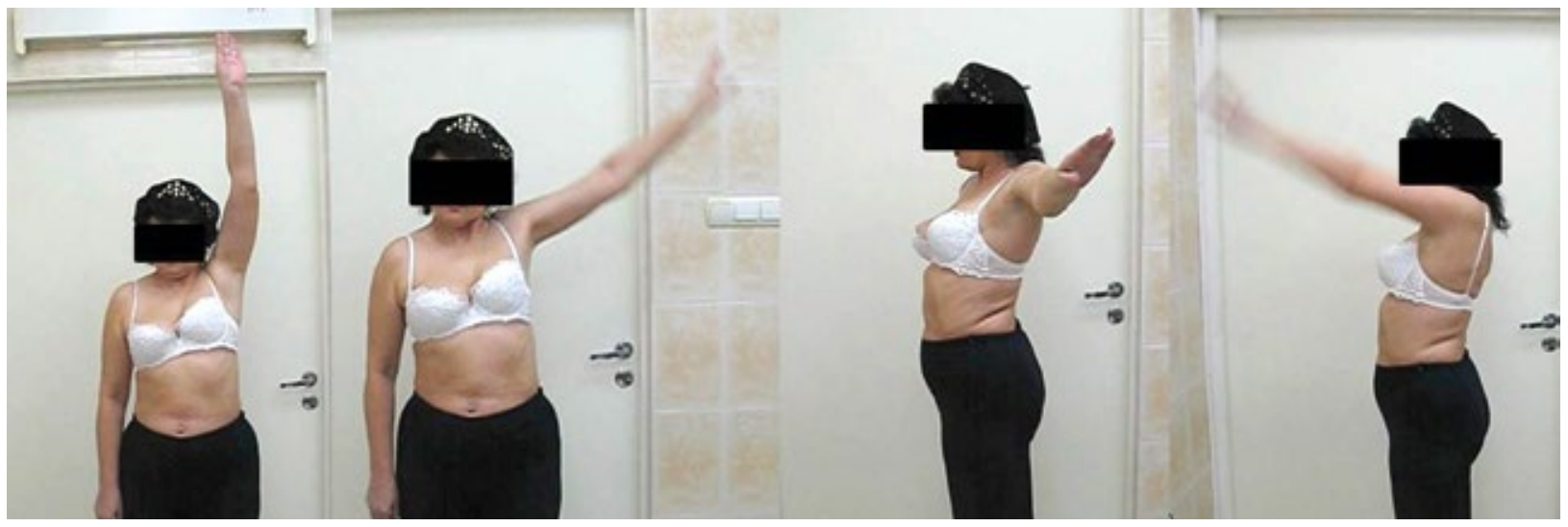

Рисунок 5. Функциональный результат через месяц после операции (при оперативном вмешательстве применен заднемедиальный доступ по Campbell -Anderssen)

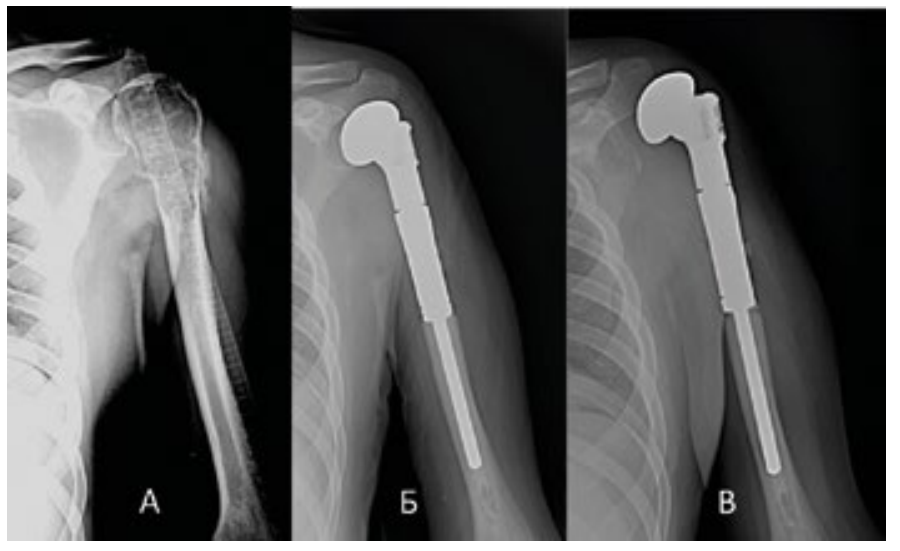

Рисунок 6. А - Рентгенограмма плечевой кости до операции (хондросаркома T2NOMO G-I Ib cт.); Б - Рентгенограмма на 3 сутки после операции (головка эндопротеза находится близко к плечевому отростку лопатки и акромион находится над ней); В - Рентгенограмма через 3 месяца после операции (головка эндопротеза смещена вверх и латерально, акромион находится сбоку медиально) 
отсутствия значимой отрицательной клинической динамики на фоне вывиха (Рис. 6).

У пациентов второй группы за период наблюдения значимых послеоперационных осложнений не выявлено.

\section{Обсуждение}

Основные цели органосохранного хирургического лечения больных с опухолевым поражением длинных костей - обеспечить максимальный радикализм во время операции и достичь адекватных функциональных и эстетических результатов с целью улучшения качества жизни больного.

Развитие и постоянное совершенствование методик органосохранного хирургического лечения в комбинации с современным анестезиологическим пособием и антибактериальной терапией в настоящее время позволяет выполнять органосохранные операции в радикальном объеме более чем у $90 \%$ больных с опухолевым поражением костей $[23,24]$. Несмотря на неоспоримые успехи, в онкоортопедии актуальной проблемой является обеспечение максимально высокого функционального статуса после операции, со снижением ограничений объема движения и приближенного к физиологическому состоянию прооперированной конечности.

По данным зарубежной литературы, широко используемые до настоящего времени АЭ плечевого сустава у больных с опухолевым поражением проксимального отдела плечевой кости имеют ряд недостатков. Так Gosheger G. в исследовании, включившем 39 пациентов, установил, что головка АЭ в большинстве случаев не кон-

\section{Список литературы:}

1. Damron T.A. Orthopedic Surgery Essentials: Onology and Basic Science. Philadelphia: Lippincott, Williams, and Wilkins; 2008.

2. Gosheger G., Gebert C., Ahrens H. et al. Endoprosthetic Reconstruction in 250 Patients with Sarcoma. Clin Orthop Relat Res. 2006; 450:164-171.

3. Некачалов В.В. Патология костей и суставов: Руководство. // СПб.: Сотис, 2000 г.

4. Aliev M.D., Teplyakov V., Sicheva L., Karpenko V. Modern orthopaedical treatment of metastatic lesion of long bones. 17th Annual Meeting of the EMSOS, Oslo, Norway, 9-11 June 2004.

5. Asavamongkolkul A, Eckardt J.J., Eilber F.R., Dorey F.J., Ward W.G., Kelly C.M., et al. Endoprosthetic reconstruction for malignant upper extremity tumors. Clin Orthop Relat Res. 1999; 360: 207-220.

6. Карпенко В.Ю. «Хирургичское лечение метастатического поражения длинных трубчатых костей как этап комбинированной терапии». Диссертация на соискание ученой степени кандидата медицинских наук. РОНЦ им. Н.Н.Блохина. Москва 2005 г.

7. Berruti A., Dogliotti L., Gorzegno G. et al. Differential Patterns of Bone Turnover in Relation to Bone Pain and Disease Extent in Bone in Cancer Patients with Skeletal Metastases. Clinical Chemistry. 1999; 45(8 Pt 1):1240-1247.

8. Iwamoto Y. Diagnosis and Treatment of Ewing's Sarcoma. Jpn J Clin Oncol. 2007; 37(2): 79-89. груэнтна суставной поверхности плечевого отростка лопатки, что, в свою очередь, приводит к значительному нарушению движения в сформированном суставе при отведении и поднятии плеча вверх и не обеспечивает необходимой стабильности [2,18]. По мнению Raiss P. использование биосинтетической манжеты фиксирующей эндопротез к лопатке увеличивает стабильность сустава и снижает риск инфекционных осложнений, но на увеличение объема движений в нем значимо не влияет [25].

Сегодня ряд зарубежных клиник применяет разработанные в последнее время "Обратные» системы модульного эндопротезирования плечевого сустава у больных с опухолевым поражением проксимального отдела плечевой кости, которые при сравнимом с АЭ количестве осложнений позволяют добиться лучших функциональных результатов. По нашим данным использование ОЭ у данной категории больных улучшает функциональные результаты после операции на 75\%, при этом в нашем исследовании после установки ОЭ осложнений не отмечено.

Ввиду ограниченного количества клинического материала и непродолжительных сроков динамического наблюдения, на настоящий момент трудно сделать категоричные заявления по успешности применения "Обратного» эндопротезирования с хирургическим заднемедиальным доступом у больных с опухолевым поражением проксимального отдела плечевой кости, однако полученные нами и другими клиниками результаты использования этой методики свидетельствуют о перспективности ее развития и дальнейшего внедрения в онкоортопедическую практику.

9. Rougraff B.T., Simon M.A., Kneisl J.S., et al. Compared with Amputation for Osteosarcoma of the Distal End of the Femur. A long-term oncological, functional, and quality-of-life study. J Bone Joint Surg Am. 1994; 76 (5): 649-656

10. Mittermayer F., Krepler P., Dominkus M., et al. Long-term followup of uncemented tumor endoprostheses for the lower extremity. Clin Orthop Relat Res. 2001; 388: 167-177.

11. Sluga M., Windhager R., Lang S., et al. Local and systemic control after ablative and limb sparing surgery in patients with osteosarcoma. Clin Orthop Relat Res. 1999; 358: 120-127.

12. Gosheger G., Goetze C., Hardes J., et al. The influence of the alloy of megaprostheses on infection rate. J Arthroplasty. 2008; 23(6): 916-920.

13. Hardes J., Ahrens H., Gebert C., et al. Lack of toxicological sideeffects in silver-coated megaprostheses in humans. Biomaterials, 2007; 28(18): 2869-2875.

14. Hardes J., von Eiff C., Streitbuerger A., et al. Reduction of periprosthetic infection with silver-coated megaprostheses in patients with bone sarcoma. J Surg Oncol. 2010: 101(5): 389-395.

15. Балберкин А.В., Шавырин Д.А. Клиническое обоснование конструкции модульной эндосистемы коленного сустава, дистального отдела бедренной кости и проксимального отдела большеберцовой кости. Саркомы костей, мягких тканей и опухоли кожи. 2011; № 4: 12-20.

16. Dieckmann R., Liem D., Gosheger G., et al. Evaluation of a reconstruction reverse shoulder for tumour surgery and tribo- 
logical comparision with an anatomical shoulder arthroplasty. Int Orthop (SICOT). 2013; 37(3): 451-456.

17. Gupta G.R., Yasko A.W., Lewis V.O., et al. Risk of local recurrence after deltoid-sparing resection for osteosarcoma of the proximal humerus. Cancer. 2009; 115(16): 3767-3773.

18. Gosheger G., Hardes J., Ahrens H., et al. Endoprosthetic replacement of the humerus combined with trapezius and latissimus dorsi transfer: a report of three patients. Arch Orthop Trauma Surg. 2005125 (1): 62-65.

19. Flury M.P., Frey P., Goldhahn J., et al. Reverse shoulder arthroplasty as a salvage procedure for failed conventional shoulder replacement due to cuff failure-midterm results. Int Orthop. 2011; 35(1): 53-60.

20. Boileau P., Watkinson D., Hatzidakis A.M., Hovorka I. Neer award 2005: the grammont reverse shoulder prosthesis: results in cuff tear arthritis, fracture sequelae, and revision arthroplasty. J Shoulder Elbow Surg. 2006; 15(5): 527-540.

References:

1. Damron T.A. Orthopedic Surgery Essentials: Onology and Basic Science. Philadelphia: Lippincott, Williams, and Wilkins; 2008.

2. Gosheger G., Gebert C., Ahrens H. et al. Endoprosthetic Reconstruction in 250 Patients with Sarcoma. Clin Orthop Relat Res. 2006; 450:164-171.

3. Nekachalov V.V. Patologiya kostei i sustavov: Rukovodstvo. // SPb.: Sotis, $2000 \mathrm{~g}$.

4. Aliev M.D., Teplyakov V., Sicheva L., Karpenko V. Modern orthopaedical treatment of metastatic lesion of long bones. 17th Annual Meeting of the EMSOS, Oslo, Norway, 9-11 June 2004.

5. Asavamongkolkul A, Eckardt J.J., Eilber F.R., Dorey F.J., Ward W.G., Kelly C.M., et al. Endoprosthetic reconstruction for malignant upper extremity tumors. Clin Orthop Relat Res. 1999; 360: 207-220.

6. Karpenko V.Yu. «Khirurgichskoe lechenie metastaticheskogo porazheniya dlinnykh trubchatykh kostei kak etap kombinirovannoi terapii». Dissertatsiya na soiskanie uchenoi stepeni kandidata meditsinskikh nauk. RONTs im. N.N.Blokhina. Moskva 2005 g.

7. Berruti A., Dogliotti L., Gorzegno G. et al. Differential Patterns of Bone Turnover in Relation to Bone Pain and Disease Extent in Bone in Cancer Patients with Skeletal Metastases. Clinical Chemistry. 1999; 45(8 Pt 1):1240-1247.

8. Iwamoto Y. Diagnosis and Treatment of Ewing's Sarcoma. Jpn J Clin Oncol. 2007; 37(2): 79-89.

9. Rougraff B.T., Simon M.A., Kneisl J.S., et al. Compared with Amputation for Osteosarcoma of the Distal End of the Femur. A long-term oncological, functional, and quality-of-life study. J Bone Joint Surg Am. 1994; 76 (5): 649-656.

10. Mittermayer F., Krepler P., Dominkus M., et al. Long-term followup of uncemented tumor endoprostheses for the lower extremity. Clin Orthop Relat Res. 2001; 388: 167-177.

11. Sluga M., Windhager R., Lang S., et al. Local and systemic control after ablative and limb sparing surgery in patients with osteosarcoma. Clin Orthop Relat Res. 1999; 358: 120-127.

12. Gosheger G., Goetze C., Hardes J., et al. The influence of the alloy of megaprostheses on infection rate. J Arthroplasty. 2008; 23(6): 916-920.

13. Hardes J., Ahrens H., Gebert C., et al. Lack of toxicological sideeffects in silver-coated megaprostheses in humans. Biomaterials, 2007; 28(18): 2869-2875.

14. Hardes J., von Eiff C., Streitbuerger A., et al. Reduction of periprosthetic infection with silver-coated megaprostheses in pa-
21. Canale T.S., Beaty J.H., Campbell's Operative Orthopaedics 12th Edition. Elsevier. 2012: 2004-2067.

22. Enneking W.F., Dunham W., Gebhardt M.C., Malawar M., Pritchard D.J. A system for the functional evaluation of reconstructive procedures after surgical treatment of tumours of the musculoskeletal system. Clin. Orthop. 1993; 286: 241-246.

23. Мачак Г.Н. «Современные возможности и перспективы комбинированного лечения остеосаркомы» автореферат диссертации на соискание ученой степени доктора медицинских наук. РОНЦ им. Н.Н.Блохина. Москва 2007г.

24. Wodajo F.M., Bickels J., Wittig J., Malawer M. Complex reconstruction in the management of extremity sarcomas. Curr Opin Oncol. 2003; 15(4): 304-312, 2003.

25. Raiss P., Kinkel S., Sauter U., et al. Replacement of the proximal humerus with MUTARS tumor endoprostheses. Eur J Surg Oncol. 20010; 36(4): 371-377.

tients with bone sarcoma. J Surg Oncol. 2010: 101(5): 389-395.

15. Balberkin A.V., Shavyrin D.A. Klinicheskoe obosnovanie konstruktsii modul'noi endosistemy kolennogo sustava, distal'nogo otdela bedrennoi kosti i proksimal'nogo otdela bol'shebertsovoi kosti. Sarkomy kostei, myagkikh tkanei i opukholi kozhi. 2011; № 4: 12-20.

16. Dieckmann R., Liem D., Gosheger G., et al. Evaluation of a reconstruction reverse shoulder for tumour surgery and tribological comparision with an anatomical shoulder arthroplasty. Int Orthop (SICOT). 2013; 37(3): 451-456.

17. Gupta G.R., Yasko A.W., Lewis V.O., et al. Risk of local recurrence after deltoid-sparing resection for osteosarcoma of the proximal humerus. Cancer. 2009; 115(16): 3767-3773.

18. Gosheger G., Hardes J., Ahrens H., et al. Endoprosthetic replacement of the humerus combined with trapezius and latissimus dorsi transfer: a report of three patients. Arch Orthop Trauma Surg. 2005125 (1): 62-65.

19. Flury M.P., Frey P., Goldhahn J., et al. Reverse shoulder arthroplasty as a salvage procedure for failed conventional shoulder replacement due to cuff failure-midterm results. Int Orthop. 2011; 35(1): 53-60.

20. Boileau P., Watkinson D., Hatzidakis A.M., Hovorka I. Neer award 2005: the grammont reverse shoulder prosthesis: results in cuff tear arthritis, fracture sequelae, and revision arthroplasty. J Shoulder Elbow Surg. 2006; 15(5): 527-540.

21. Canale T.S., Beaty J.H., Campbell's Operative Orthopaedics 12th Edition. Elsevier. 2012: 2004-2067.

22. Enneking W.F., Dunham W., Gebhardt M.C., Malawar M., Pritchard D.J. A system for the functional evaluation of reconstructive procedures after surgical treatment of tumours of the musculoskeletal system. Clin. Orthop. 1993; 286: 241-246.

23. Machak G.N. "Sovremennye vozmozhnosti i perspektivy kombinirovannogo lecheniya osteosarkomy» avtoreferat dissertatsii na soiskanie uchenoi stepeni doktora meditsinskikh nauk. RONTs im. N.N.Blokhina. Moskva 2007g.

24. Wodajo F.M., Bickels J., Wittig J., Malawer M. Complex reconstruction in the management of extremity sarcomas. Curr Opin Oncol. 2003; 15(4): 304-312, 2003.

25. Raiss P., Kinkel S., Sauter U., et al. Replacement of the proximal humerus with MUTARS tumor endoprostheses. Eur J Surg Oncol. 20010; 36(4): 371-377. 
Информация об авторах:

1. Карпенко Вадим Юрьевич, к.м.Н., старший научный сотрудник группы опухолей костей и мягких тканей, отдела хирургического лечения опухолей центральной нервной и костно-мышечной систем МНИОИ им. П.А. Герцена - филиал ФГБУ «НМИРЦ» Минздрава России

2. Державин Виталий Андреевич, к.м.н., научный сотрудник группы опухолей костей и мягких тканей, отдела хирургического лечения опухолей центральной нервной и костномышечной систем МНИОИ им. П.А. Герцена - филиал ФГБУ «НМИРЦ» Минздрава России

3. Бухаров Артем Викторович, к.м.н., старший научный сотрудник группы опухолей костей и мягких тканей, отдела хирургического лечения опухолей центральной нервной и костно-мышечной систем МНИОИ им. П.А. Герцена - филиал ФГБУ «НМИРЦ» Минздрава России
Information about authors:

1. Karpenko Vadim - PhD, senior researcher of group of bone and soft tissue tumors, division of surgical treatment of tumors of the central nervous and musculoskeletal systems P. Hertsen MORI - branch of NMRRC

2. Derzhavin Vitaly - PhD, senior researcher of group of bone and soft tissue tumors, division of surgical treatment of tumors of the central nervous and musculoskeletal systems P. Hertsen MORI - branch of NMRRC

3. Buharov Artem - PhD, senior researcher of group of bone and soft tissue tumors, division of surgical treatment of tumors of the central nervous and musculoskeletal systems P. Hertsen MORI - branch of NMRRC

\section{Оформление ссылки для цитирования статьи:}

Карпенко В.Ю., Державин В.А., Бухаров А.В. Эндопротезирование плечевого сустава при опухолевом поражении проксимального отдела плечевой кости. Исследования и практика в медицине. 2015; 2(2): 15-23. DOI: 10.17709/2409-2231-2015-2-2-15-23

Karpenko V.Yu., Derzhavin V.A., Buharov A.V. Endoprosthetic replacement in patients with tumors of proximal humerus. Issled. prakt. Med. 2015; 2(2): 15-23. DOI: 10.17709/2409-2231-2015-2-2-15-23

Конфликт интересов. Все авторы сообщают об отсутствии конфликта интересов.

Conflict of interest\& All authors report no conflict of interest. 